\title{
COVID-19 e atenção a pessoas com deficiência e grupos especiais na clínica-escola de Odontologia
}

\author{
Alexandre Franco Miranda*; Andréia de Aquino Marsiglio**; Daniele Machado da Silveira**; Rafaela \\ Sabino e Andrade**; Taia Maria Berto Rezende***; Lais David Amaral****; Claudia Maria de Souza \\ Peruchi****; Eric Jacomino Franco****
}

* Professor Doutor, Curso de Odontologia e Programa de Pós-graduação em Gerontologia, Universidade Católica de Brasília

** Professora Mestre, Curso de Odontologia, Universidade Católica de Brasília

*** Professora Doutora, Curso de Odontologia e Programa de Pós-graduação em Ciências Genômicas e Biotecnologia, Universidade Católica de Brasília; Pesquisadora colaboradora sênior do Programa de Pós-graduação em Ciências da Saúde, Universidade de Brasília

**** Professor(a) Doutor(a), Curso de Odontologia, Universidade Católica de Brasília

Recebido: 11/06/2020. Aprovado: 23/02/2021.

\begin{abstract}
RESUMO
O presente artigo tem como finalidade abordar orientações e sugestões para o atendimento odontológico de pacientes com necessidades especiais (pessoas com deficiência) e idosos nas clínicas de graduação, considerando as implicações da COVID-19. A partir da publicação de orientações nacionais e internacionais de biossegurança, cuidados em saúde bucal e condutas odontológicas, foram elaboradas diretrizes para esta abordagem clínica. Espera-se a disseminação do conhecimento, informações e adaptações necessárias no ensino para uma melhor assistência e contribuição educacional aos graduandos, além da realização das atividades práticas de maneira segura no ambiente universitário.

Descritores: Infecções por Coronavírus. Pessoas com Deficiência. Odontologia Geriátrica. Educação em Odontologia. Desenvolvimento de Pessoal.
\end{abstract}

\section{INTRODUÇÃO}

A formação do cirurgião-dentista ainda apresenta lacunas na capacitação do egresso para atuar clinicamente frente às possíveis adversidades e condições diferenciadas associadas à assistência a pessoas com deficiência e grupos especiais ${ }^{1,2}$. As atividades de ensino devem contemplar orientações e capacitação mínima para a prática de Odontologia para pacientes com necessidades especiais (OPNE), que inclui pessoas com deficiência, grupos especiais e idosos (Odontogeriatria), a partir de uma formação generalista, reflexiva, humana, empática, interdisciplinar e de avaliação do paciente de maneira integral e individualizada ${ }^{1,3-5}$.

A OPNE ainda é marginalizada e pouco acessível, principalmente pela escassez de 
profissionais capacitados e de estratégias educacionais que valorizem esse tipo de prática clínica na graduação; situação agravada pela pandemia associada à coronavirus disease $(\text { COVID-19) })^{6,7}$.

O presente artigo tem por objetivo apresentar as diretrizes adotadas e orientações práticas elaboradas pela equipe docente da disciplina obrigatória de Práticas Clínicas Integradas para Pessoas com Deficiência e Grupos Especiais (Clínica de Odontologia para Pacientes Especiais) do curso de Odontologia da Universidade Católica de Brasília (UCB), para a realização de atendimentos ambulatoriais seguros, além de medidas preventivas práticas.

\section{RELATO DE EXPERIÊNCIA}

$\mathrm{O}$ projeto pedagógico do curso de Odontologia da UCB baseia-se na concretização de um modelo pedagógico diferenciado, cujo principal intento é a formação integral e ajustada às necessidades da sociedade brasileira, em especial no que diz respeito ao sistema de saúde, priorizando iniciativas de promoção da saúde individual e da comunidade ${ }^{1}$.

A percepção interdisciplinar da condição de deficiência e/ou necessidades especiais proporcionam aos estudantes e equipe docente um processo de aprendizagem com o foco nas relações humanas, afetivas, emocionais, biológicas, associadas às condições sociais, culturais, familiares, cristãs e da coletividade ${ }^{1}$.

Atividades educacionais com o foco na assistência em saúde são abordadas desde o primeiro ano do curso, disciplinas optativas como Libras e obrigatórias como a prática clínica direcionada a pessoas com deficiência e grupos especiais nos ciclos da vida fazem parte da matriz curricular do curso ${ }^{8}$.

\section{Práticas clínicas integradas para pessoas com} deficiência e grupos especiais - clínica de

\section{Odontologia para pacientes especiais}

Trata-se de disciplina obrigatória, de caráter prático (associado a atividades teóricas, demonstrativas e científicas), organizada em 80 horas de atividade, com 8 docentes e 30 duplas de estudantes por semestre, com a finalidade de proporcionar a vivência de discentes e docentes a partir de experiências que envolvam os aspectos educacionais, sociais, éticos e clínicos da atenção a pacientes com necessidades especiais e idosos, respeitando sempre as limitações e especificidades de cada indivíduo ${ }^{1,6}$.

Existe a integração e aprendizado comum dos estudantes e professores com as mais diversas condições de dificuldades, adversidades, adaptações e manejo clínico de pacientes, proporcionando maior participação, compartilhamento das decisões e condutas, além da motivação em poder contribuir com grupos marginalizados da sociedade e com poucos recursos financeiros.

A proximidade da relação discente, pessoa com deficiência e/ou pertencente a algum grupo de necessidades especiais, família e docente contribui para momentos de afeto e carinho expressos por meio de abraços, apertos de mãos, beijos, atividades interativas e clínicas mais próximas que interferem positivamente para o correto manejo e adaptação profissional-paciente. A partir dessa nova realidade da prática odontológica associada à COVID-19, ainda não foi estabelecido de maneira efetiva como serão os atendimentos desses pacientes, bem como a correta estrutura logística e temporal na realização dos atendimentos odontológicos na universidade $\mathrm{f}^{6,7,9}$.

Desta forma, diretrizes com breves orientações sobre como proceder diante desse novo cenário do ensino e práticas odontológicas foram elaboradas. Os professores da disciplina se reuniram virtualmente para discutir e elaboração da melhor estratégia e busca na literatura nacional e internacional de informações atualizadas para a prática odontológica, de maneira geral e 
relacionada aos pacientes com deficiência e grupos especiais durante esse período de pandemia. Posteriormente, os principais temas relevantes a serem abordados nas atividades práticas foram organizados em tópicos, com o intuito de facilitar a compreensão dos alunos, pacientes, familiares, corpo docente e equipe auxiliar.

Foi realizada uma análise dos principais guias de orientações, manuais e artigos científicos publicados em português e inglês, de órgãos competentes nacionais, entidades reconhecidas mundialmente na assistência odontológica em geral e de pessoas com necessidades especiais e idosos, além de artigos publicados em periódico de referência nacional no ensino e práticas educacionais na Odontologia.

Foram selecionados 19 documentos considerados mais relevantes à prática clínica e ao atendimento ao paciente com necessidades especiais nos ciclos da vida, associado à pandemia da COVID-19. A escrita foi compartilhada pelos docentes da disciplina, que fizeram as suas sugestões, análises críticas e modificações para a concretização dessas orientações.

\section{A relação da COVID-19 com o ensino da prática odontológica dedicada a grupos especiais}

A pandemia COVID-19 interferiu diretamente nos serviços educacionais, em especial nas atividades práticas da assistência odontológica nas instituições públicas e privadas. O distanciamento social permitiu apenas atividades remotas no ensino e grupos virtuais de estudos, trazendo a necessidade de reestruturação logística e de planejamentos de atividades futuras $^{6,11}$.

O indivíduo infectado pelo coronavírus apresenta sintomas como tosse, febre e dores de cabeça, porém pode ser assintomático ${ }^{9,10}$. Durante a prática odontológica existe grande risco de infecção cruzada, potencializada pelos aerossóis associados aos procedimentos e pela elevada carga viral presente nas vias aéreas superiores de pacientes contaminados ${ }^{10-12}$.

A clínica-escola do curso de Odontologia da UCB presta assistência a pessoas com deficiência e grupos especiais nos diferentes ciclos da vida (criança, adolescente, adulto e idoso), bem como um suporte educacional de saúde bucal aos familiares e cuidadores. As atividades clínicas realizadas pelos alunos têm o foco no atendimento generalista, o que envolve todas as áreas regulamentadas na formação profissional.

Desta forma, as diretrizes e protocolos adotados na assistência odontológica de pacientes especiais são baseados nas decisões das demais áreas do curso, como Educação em Saúde, Saúde Coletiva, Aspectos Éticos e Legais da Prática Odontológica, Periodontia, Dentística, Prótese Dentária, Estomatologia, Cirurgia Bucal, Endodontia, Odontopediatria, Odontogeriatria e Estágios Supervisionados em Odontologia (Práticas Clínicas Integradas).

Os pacientes assistidos são pessoas com necessidades especiais das mais variadas idades (bebê ao idoso), considerados indivíduos mais vulneráveis, fragilizados e susceptíveis a contaminações. Por isso, além do correto cumprimento das normas de biossegurança para qualquer atendimento clínico, traz a exigência de atenção diferenciada, com condutas educativas e preventivas, principalmente quanto à realização de procedimentos odontológicos considerados minimamente invasivos nesse momento de pandemia, de maneira a evitar a disseminação viral, contaminação desses indivíduos e seus familiares. É importante ressaltar que os cuidados em saúde e normas de biossegurança ${ }^{11,13}$ devem ser direcionados a todos os envolvidos durante $\mathrm{o}$ atendimento odontológico especial.

No entanto, alguns aspectos, principalmente, no que se refere ao relacionamento e proximidade com os pacientes especiais serão desafios a 
enfrentarmos juntos nesse novo contexto de atuação profissional e educacional (docentediscente) durante e após a pandemia. Devido às diversas possibilidades de atuação profissional, bem como personalidades e condições físicas, mentais e sistêmicas diferenciadas dos pacientes, pretende-se desenvolver, aos poucos e com segurança, as melhores estratégias de manejo clínico, adaptação profissional e ações odontológicas de acordo com as principais necessidades dos pacientes a partir da capacitação e interação docente-discente.

Vivencia-se um momento único de renovações nas atividades odontológicas dedicadas aos pacientes especiais e seus familiares. $\mathrm{O}$ caloroso abraço de boas vindas e a proximidade existente nos vínculos afetivos no atendimento odontológico especial terão que aguardar o melhor momento para as relações pessoais de contato. Mudanças específicas são necessárias para a realização, de forma segura, das atividades educacionais e clínicas para esses pacientes, contribuindo diretamente para a promoção da saúde e qualidade de vida, por meio da formação e vivências discentes na universidade.

\section{Condutas para recepção e sala de espera no atendimento odontológico especial}

As orientações para triagem prévia e agendamento ${ }^{6,7,12-15}$ e para a recepção e sala de espera $^{6-8,13,14,16}$ constam dos quadros 1 e 2, respectivamente.

Quadro 1. Instruções para triagem prévia e agendamento

Triagem prévia do paciente e seus familiares (ligação telefônica ou WhatsApp) (6,14 $^{\text {(1) }}$

Agendamentos com horários definidos e espaçados para garantir o distanciamento adequado ${ }^{7,11,13}$

Orientação ao responsável pelo paciente da presença de apenas um acompanhante (quando possível), no dia do atendimento ${ }^{12-15}$

Quadro 2. Instruções para recepção e sala de espera

Repetição da triagem prévia, contemplando o paciente e seus familiares ${ }^{6,8,14}$

Manutenção do distanciamento entre os assentos para os pacientes na sala de espera ${ }^{8,13,14}$

Orientação dos pacientes e seus acompanhantes sobre as medidas de proteção e higiene respiratória após a tosse $\mathrm{e}^{6,7,14,16}$

Manutenção dos ambientes ventilados, com portas e janelas abertas ${ }^{13,14}$

Orientação ao paciente e cuidador para higienizar as mãos com água e sabão líquido ou gel de álcool $70 \%$ (glicerinado), logo que chegar $6,7,8,14,16$

Instruir o responsável e o paciente para evitar transitar pela sala de espera e não tocar objetos que não são sejam essenciais ${ }^{6,8,13}$

Evitar o contato físico caloroso ao receber o paciente especial e seus familiares, porém sem a perda da simpatia, empatia e humanização no contato com o paciente especial ${ }^{6,7,13,14}$

Uso obrigatório de máscaras pelo paciente e seu cuidador.

*o uso de máscaras não será obrigatório para pacientes que não possuem um perfil cognitivo e/ou de colaboração para o correto entendimento, pacientes respiradores bucais e, associados ao quadro de babação ${ }^{6,14}$

Orientação aos cuidadores para utilização de lenços de papel descartáveis para secagem da saliva do paciente especial (se necessário) e higienização das mãos sempre após contatos físicos, conforme padronizações estipuladas pelas atividades de biossegurança do curso (lavagem correta das mãos com água e sabão; utilização de álcool $70 \%)^{6,8,14,16}$ 


\begin{abstract}
Diretrizes e orientações no período de odontológico de pessoas com deficiência e grupos pandemia da COVID-19

As orientações para atendimento COVID-19 constam do quadro 3.
\end{abstract}

Quadro 3. Instruções para atendimento odontológico de pessoas com deficiência e grupos especiais

Utilização de fotografias dos discentes e docentes nos jalecos descartáveis para reconhecimento dos profissionais por parte dos pacientes especiais e familiares ${ }^{7,10,11}$

Recepção do paciente especial sem estar paramentado e cumprimentá-lo, mantendo distância mínima de 2 metros, evitando qualquer contato físico ${ }^{7,10,11}$

Realização de diálogo franco e amistoso com o paciente especial e responsáveis, para condicioná-los às mudanças relacionadas a biossegurança, bem como o novo perfil de relacionamento profissional (discente/docente) durante as atividades clínicas ${ }^{7,10,11}$

Reforço da comunicação com paciente e responsável, após o atendimento clínico e correta desparamentação do discente ou docente ${ }^{7,10,11}$

Confirmar se o paciente pode ser submetido ao tratamento odontológico indicado e indicar ao responsável/cuidador, a área onde se deve remover e deixar objetos pessoais ${ }^{7,10,11}$

Incentivar o uso da tecnologia digital para envio de fotos, vídeos explicativos ou realização de chamadas por vídeo ${ }^{7,10,11}$

Informar os responsáveis e pacientes sobre quaisquer mudanças ocorridas e exigidas na consulta odontológica por meio de WhatsApp na forma de uma lista de orientações, por meio de folder explicativo ou até por vídeos informativos ${ }^{7,10,11}$

Quando necessária, a presença do responsável ou familiar no atendimento clínico, esta será condicionada a paramentação (uso de gorro) e uso de máscara. Este deverá permanecer a uma distância segura do box de atendimento e não deverá usar o celular ${ }^{7,10,11}$

\section{Recomendações para a correta prática odontológica em época de pandemia}

Considerando que os cirurgiões-dentistas, alunos de graduação e professores que desempenham atividades nas clínicas integradas atendendo pacientes estão entre os profissionais com alto risco de contaminação pelo vírus que causa a COVID-19, orienta-se que, durante a pandemia, sejam realizados somente os procedimentos essenciais e os considerados de urgência e emergência ${ }^{9,10,13}$.

São consideradas

emergências odontológicas: hemorragia, celulite difusa intra ou extraoral e traumas com potencial de comprometimento de vias aéreas. Estes casos, serão orientados a procurar atendimento hospitalar. Por outro lado, são consideradas urgências odontológicas: dores de origem endodôntica (pulpites, abscessos), traumática (traumatismo dental), periodontal (abscessos gengivais ou periodontais, gengivites e periodontites ulcerativas, pericoronarite) ou cirúrgicas (alveolite, remoção de suturas); necessidades de biópsia de lesões com suspeita de malignidade e necessidade de adequação bucal para pacientes oncológicos ou pacientes pré-transplantes de órgãos sólidos. Estes casos, poderão ser atendidos no ambiente de clínica-escola $^{14,17-19}$.

No entanto, tratando-se de pacientes com necessidades especiais e idosos em geral, as definições de urgência e emergência, seus respectivos protocolos para solucionar ou minimizar cada um desses casos, nem sempre são aplicáveis na prática clínica. Desta forma, diante 
das várias condições de deficiência e grupos especiais existentes, não existe uma correta normativa e determinação do que deve ou não ser realizado clinicamente. $\mathrm{O}$ profissional (discente sob suporte educacional docente) em conjunto com a família, a partir de planejamentos interdisciplinares, determinarão a melhor estratégia de ação odontológica por meio da decisão compartilhada ${ }^{10,14,19}$.

\section{Desinfecção dos dispositivos de estabilização protetora, conforto para o paciente especial e adaptação profissional no atendimento odontológico}

Estratégias que contribuem para maior aceitação do tratamento odontológico devem ser priorizadas, bem como promover maior conforto e tranquilidade ao paciente especial como travesseiros, suporte de pescoço e encosto de cabeça, disponíveis nas nossas atividades clínicas.
As estabilizações protetoras (faixas estabilizadoras) são indicadas para pacientes pouco ou não colaborativos, com o objetivo de proteger o paciente e a equipe de trabalho (discentes e docente) de possíveis injúrias durante a realização de procedimentos odontológicos. Outro recurso utilizado para o atendimento odontológico de pacientes com deficiência e grupos especiais, disponível nas nossas atividades clínicas, é o colchão a vácuo, que proporciona maior conforto, estabilidade corporal e segurança aos pacientes durante o tratamento, além de contribuir como uma efetiva estratégia nas atividades lúdicas ${ }^{11,16}$.

Considerando que as clínicas integradas e equipos odontológicos passarão por um rigoroso processo de desinfecção realizado pelas equipes de limpeza da universidade antes e após os atendimentos, os cuidados necessários ao correto uso e desinfecção dos dispositivos utilizados na clínica estão agrupados no quadro 4.

Quadro 4. Instruções para correto uso e desinfecção dos dispositivos utilizados na clínica odontológica de pessoas com deficiência e grupos especiais

Orientação aos alunos e professores para não solicitarem aos responsáveis, lençóis para estabilização protetora do paciente especial, bem como evitar a presença de brinquedos ${ }^{7,10}$

*em caso de extrema necessidade, todo o processo de higienização (água corrente + sabão) e desinfecção (álcool 70\%) deve ser realizado em cada atendimento

Faixas de estabilização protetora de material sintético deverão ser desinfetadas com álcool $70 \%$ antes e após sua utilização ${ }^{10}$

*estas não serão reutilizadas em outro paciente no mesmo período de atendimento

Colchões à vácuo, almofadas posicionadoras de material sintético, travesseiros e o suporte para a cabeça também serão desinfetadas com álcool $70 \%$ antes e após sua utilização ${ }^{10,12,13,16}$

Abridores de boca confeccionados (palitos de madeira + esparadrapo + gaze) deverão ser confeccionados com antecedência, autoclavados antes do uso e descartados em seguida ${ }^{10}$

Abridores de boca comercializados deverão ser submetidos aos protocolos e normas de biossegurança Central de Material e Esterilização do curso de Odontologia para posterior reutilização, quando necessário $^{10}$

\section{CONSIDERAÇÕES FINAIS}

A partir da percepção educacional dos autores, espera-se que as orientações possam servir como norteadoras para a continuidade dessa prática clínica durante a graduação com o foco na desmistificação do atendimento 
odontológico para pessoas com deficiência e grupos especiais nos ciclos da vida.

Estas atividades deverão ser reorganizadas com base nas orientações, diretrizes e condutas que visam a não transmissão cruzada e diminuição da contaminação pelo coronavírus, a partir da realidade institucional de cada curso.

\section{ABSTRACT \\ COVID-19 and assistance to people with disabilities and special groups in dental school clinic}

The aim of the current article is to address the guidelines and suggestions for dental care of patients with special needs (people with disabilities) and elderly in undergraduate dental clinics by taking into account the impacts caused by the COVID-19 pandemic. Guidelines for the Special Care Dentistry clinical approach were developed based on the publication of national and international guidelines on biosafety, oral health care, and dental procedures. Knowledge and information promotion, and the necessary adaptations in teaching are expected to allow better assistance and educational contribution to undergraduate students, in addition to performing practical activities in a safer way in college environment.

Descriptors: Coronavirus Infections. Disabled Persons. Geriatric Dentistry. Education, Dental. Staff Development.

\section{REFERÊNCIAS}

1. Cruvinel VRN, Franco EJ, Bezerra L, Alves MM, Miranda AF, Carvalho DR. A formação do cirurgião-dentista generalista na Universidade Católica de Brasília. Rev ABENO. 2010; 10(2):12-9.

2. Da Silva TD, Santaella NG, Caminha RDG, Santos PSSS. Percepção de estudantes de graduação sobre a importância da disciplina Odontologia para Pacientes com Necessidades Especiais. Rev ABENO. 2020; 20(1):26-32.

3. Penha ES, Tenório DA, Fonseca RA,
Guênes GMT, Montagna E. Caracterização do componente curricular Odontologia para Pacientes com Necessidades Especiais. Rev ABENO. 2018; 18(2):13-9.

4. Santos MFS, Hora IAA. Atenção odontológica a pacientes especiais: atitudes e percepções de acadêmicos de odontologia. Rev ABENO. 2012; 12(2):207-12.

5. Jacomine JC, Ferreira R, Sant'Ana ACP, Rezende MLR, Greghi SLA, Damante CA, Zangrando MSR. Saúde bucal e Pacientes com Necessidades Especiais: percepções de graduandos em Odontologia da FOB-USP. Rev ABENO. 2018; 18(2):45-54.

6. Beetstra S. Special care dentistry in the world of COVID-19. Spec Care Dentist. 2020; 40:215.

7. Brookes V, Rafique. Recommendations for Special Care Dentistry during COVID-19 pandemic. British Society for Gerodontology. the British Society for Disability and Oral Health and the Faculty of Dental Surgery. Royal College of Surgeons of England. 2020. Volume 1. 5p. [Acesso em: 15 de junho de 2020]. Disponível em: file:///Users/special/Downloads/Special\%20C are\%20Dentistry\%20COVID19\%20(1).pdf

8. Universidade Católica de Brasília. Curso de Odontologia. Matriz Curricular 2020 Período Integral. [Acesso em: 29 de julho de 2020]. Disponível em: https://ucb.catolica. edu.br/portal/wp-content/uploads/2019/02/ Matriz-integral-Odontologia.pdf.

9. Coulthard P. Dentistry and coronavirus (COVID-19) - moral decision-making. $\mathrm{Br}$ Dent J. 2020; 228(7):503-5.

10. Franco JB. et al. Cuidados odontológicos na era do COVID - 19: recomendações para procedimentos odontológicos e profissionais. Rev Assoc Paul Cir Dent. 2020; 74(1):18-21.

11. Franco EJ, Da Silveira DM, Lima SMF, 
Azevedo TDPL, Arruda MP, Amaral LD, Dantas EMGL, Tavares MG, Rezende TMB, Santos GAGRM, Nery DTF, Miranda AF, De Paula DS. Protocolo de Biossegurança em Odontologia [recurso eletrônico]: COVID-19. Brasília-DF, Universidade Católica de Brasília. Volume 1. 38p. ISBN 978-65-87629-00-1. [Acesso em: 29 de julho de 2020]. Disponível em: http://ucb. catolica.edu.br.

12. Brasil. Agência Nacional de Vigilância Sanitária (ANVISA). Uso de Equipamento de Proteção Individual - EPI e Isolamento. 2020.

13. Conselho Federal de Odontologia (CFO). Manual de boas práticas em biossegurança para ambientes odontológicos. Rio de Janeiro, 2020.

14. Nebraska Dental Association. American Dental Association (ADA). Interim guidance for minimizing risk of COVID-19 transmission, 2020. [Acesso em: 11 de julho de 2020]. Disponível em: https://www. nedental.org/docs/librariesprovider32/defau lt-document-library/nda-dental-officeopening-protocols.pdf?sfvrsn=0.

15. American Dental Association (ADA). ADA News Archives. ADA Adds frequently asked questions from dentists to coronavirus resources. Chicago, 12 março 2020 [Acesso em: 18 de julho de 2020]. Disponível em: https://www.ada.org/en/publications/adanews/2020-archive/march/ada-addsfrequently-asked-questions-from-dentiststo-coronavirus-resources.
16. Brasil. Agência Nacional de Vigilância Sanitária (ANVISA). Procedimento: Limpeza e Desinfecção de ambientes, equipamentos, utensílios potencialmente contaminados, gerenciamento de resíduos sólidos e efluentes sanitários. 2020.

17. Ather A, Patel B, Ruparel NB, Diogenes A, Hargreaves KM. Coronavirus disease 19 (COVID-19): implications for clinical dental care. J Endod. 2020; 46(5):584-95.

18. American Dental Association (ADA). Summary of ADA guidance during the COVID-19 crisis. Chicago, 01 abril 2020 [Acesso em: 10 julho 2020]. Disponível em: https://success.ada.org/ /media/CPS/Files/ COVID/COVID-19_Int_Guidance_Sum mary.pdf?utm_source=adaorg\&utm_mediu $\mathrm{m}=$ covid-resources-lp\&utm_content $=\mathrm{cv}-$ pm-summary-guidance\&utm_campaign $=$ covid-19\& ga=2.153317240.1307346859. 1585573040-13458067.1578068990?utm source=adaorg\&utm_medium=adanews\&ut m_content=cv-pm-summary-guidance \&utm campaign=covid-19.

19. American Dental Association (ADA). ADA News Archives. ADA develops guidance on dental emergency, nonemergency care. Chicago, 18 março 2020 [Acesso em: 08 julho 2020]. Disponível em: https://www.ada. org/en/publications/adanews/2020-archive /march/ada-developsguidance-on-dental-emergencynonemergency-care.

\section{Correspondência para:}

Alexandre Franco Miranda

e-mail: alexandref@ucb.br

Curso de Odontologia - UCB

Bloco S - Campus I - QS 07 Lote 01 - Bloco S -

EPCT, Águas Claras

71966-700 - Taguatinga/DF 